psychopraxis. neuropraxis $2022 \cdot 25: 103-106$ https://doi.org/10.1007/s00739-022-00783-y Angenommen: 13. Januar 2022 Online publiziert: 2. Februar 2022

(c) Der/die Autor(en) 2022

\section{Steinmair ${ }^{1,2,3} \cdot$ N. Masic ${ }^{1}$ D. Schwigon ${ }^{1} \cdot$ H. Löffler-Stastka}

${ }^{1}$ Klinik für Psychoanalyse und Psychotherapie, Medizinische Universität Wien, Wien, Österreich

${ }^{2}$ Karl Landsteiner Privatuniversität für Gesundheitswissenschaften, Krems, Österreich

${ }^{3}$ Klinische Abteilung für Augenheilkunde, Universitätsklinikum St. Pölten, St. Pölten, Österreich

\title{
Affektregulation und soziale Kognition bei Psychose
}

\section{Einleitung}

Die Art und Weise wie Informationen in sozialen Kontexten verarbeitet, erinnert und angewandt werden, wird als soziale Kognition bezeichnet [1]. Mentale Prozesse, die der sozialen Wahrnehmung, der sozialen Urteilsbildung und der sozialen Einflussnahme dienen, sind Forschungsgegenstand im Themenschwerpunkt soziale Kognition [2]. Soziale Kognition ermöglicht es, Verhalten, Emotionen und Gedanken anderer Menschen $\mathrm{zu}$ verstehen und darauf $\mathrm{zu}$ reagieren.

\section{》) Psychotische Erfahrungen} gehen mit Einschränkungen der sozialen Kognition einher

Die Interpretation sozialer Ereignisse geschieht subjektiv; aus Interaktionen mit der Umgebung entstehen mit der Zeit mentale Strukturen und Schemata, welche wiederum mentale Prozesse beeinflussen. Psychotische Erfahrungen gehen mit Einschränkungen der sozialen Kognition einher [3]. Das Entstehen psychotischer Zustände ist in synergetischen Modellen und folgend einem energetischen Verständnis von Emotionen die Folge des Erreichens kritischer Level von emotionaler Spannung [4]. In einer aktuellen Metaanalyse zeigte sich eine moderate messbare Assoziation zwischen psychotischen Symptomen und sozialer Kognition [3]. Die Subanalyse zeigte vor allem für "theory of mind“ (i.e., Mentalisierungsfähigkeit: $r=-0,349)$ und Emotionserkennung $(r=-0,334)$ moderate Effekte, wohingegen sich für soziale Wahrnehmung,
Emotionsregulation und Attributionsverzerrungen kleine Effekte nachweisen ließen $(r=-0,188 ; r=-0,169 ; r=-0,143)$.

Neuropsychologische Defizite sind bei Menschen mit erhöhtem Risiko für eine Psychose oft bereits vorhanden und zählen zu jenen Faktoren, welche die Vulnerabilität für die Manifestation einer Psychose erhöhen [5] und das Zurechtkommen in der realen Welt prädizieren [6]. Soziale Kognition wurde als grundlegender Mediator identifiziert, welcher den Zusammenhang zwischen Neurokognition und funktionalem Outcome vermittelt [5]. Vor allem die Negativsymptomatik (Affektverflachung, Alogie, Avolition, Asozialität, Anhedonie) zeigte sich für den Zusammenhang zwischen Kognition und Outcome verantwortlich [6].

\section{Fallvignette}

„Ich werde eine Universität gründen. Eine neue Universität für diese Fische“ (zeigt eine Packung Fisherman's Friend Pastillen [Produzent: CFP Brands Süßwarenhandels $\mathrm{GmbH} \& \mathrm{Co}$. KG, Bonn, Deutschland; Orig. Hersteller: Lofthouse of Fleetwood Ltd., Fleetwood (Lancashire), Großbritannien]). Riecht an der Packung. „Dieser Fisch ist verdorben. Ich werde mich beklagen. Sie gehören mir, sie werden mir jetzt den Brief ... ich diktiere Ihnen jetzt den Brief. Schreiben Sie." Als die Explorierende der Aufforderung nicht Folge leistet, wirkt Frau C sichtlich aufgebracht. Auf die Frage, woher sie denn wisse, dass diese Pastillen vergiftet sind, erwidert Frau C, dass der Geschmack abscheulich sei und behauptet außerdem, die Erfinderin der Pastillen zu sein. Wenn sie glaube, die
Erfinderin zu sein, warum sie dann nicht einfach den Geschmack geändert habe? Frau C wird auf diesen Einwurf der Explorierenden sichtlich unruhiger und beginnt hastig ihr Gesicht zu betasten. Im Verlauf des weiteren Gesprächs steht sie auf, möchte den Raum verlassen kommt zwar wieder zurück und nimmt auf dem Sessel Platz, bleibt aber in steter Bewegung. Nach einer Weile des Schweigens stellt sie, ihr Gesicht betastend fest, dass es nur halb da sei. Wenig später stellt sie fest, es fühle sich nun „ganz weg“ an. Es sei „voller Rogen, fauler Fischrogen“. Blickkontakt zur Explorierenden ist nun nicht mehr möglich. Wie um das (nicht mehr vorhandene) Gesicht zu verstecken, hält Frau C den Kopf zu Boden gesenkt. Frau $\mathrm{C}$ fängt unvermittelt an, in ihrer Tasche herumzuwühlen, bis sie schließlich beginnt, ihr eigenes Gesicht abzutasten und $\mathrm{zu}$ manipulieren. „Ich muss die Rogen zerschneiden!“ Die Gesprächssequenz endet damit, dass Frau C mit einem Aufschrei feststellt „Ich rede nicht mehr mit Ihnen! Ich habe Sie jetzt gekündigt!" und die Explorierende auffordert, sofort den Raum zu verlassen. Als die Explorierende der Aufforderung nicht folgt, sagt Frau C, mehr zu sich selbst als zum Gegenüber: „Sie sind entlausend! Entlaust!“

\section{Diskussion}

In dieser Episode aus der Klinik zeigt sich einerseits die Herausforderung der Gestaltung einer therapeutischen Beziehung mit PatientInnen mit akutem Beginn einer Psychose, andererseits wird deutlich, dass die Art und Weise, wie Affektregulation passiert, therapeutische 
Intervention schwierig, aber umso notwendiger macht. Was sich der Explorierenden zunächst als verbales Ausagieren und leichte psychomotorische Unruhe zeigt, endet schließlich in einer verbalen Attacke. Bereits in Ciompis Affektlogik, welche die Interaktion von Kognition und Affekt erforscht, ist der energetische Aspekt von Affekten entscheidend [4]. Emotionale Phänomene wurzeln demnach in bioenergetischen $\mathrm{Zu}$ ständen, welche motorische und kognitive Phänomene entweder motivieren oder auch blockieren können. Der Einfluss von Affekten auf kognitive Prozesse besteht damit zum Teil in einer, die Aufmerksamkeit lenkenden, Filterfunktion.

Das „Prinzip der freien Energie“ von Friston besagt, dass biologische Systeme, also auch das menschliche Gehirn, kontinuierlich daran arbeiten, „freie Energie“ (Überraschung) zu vermeiden. Demnach gilt es Diskrepanzen zwischen Annahmen über die Realität und Wahrnehmung zu verarbeiten und weitgehend zu vermeiden. Diese Diskrepanzen entsprechen freier Energie, im Minimieren dieser freien Energie passiert eine Anpassung an die Umgebung des Systems, mit dem Ziel, Überraschung und Unsicherheit $\mathrm{zu}$ reduzieren [7]. Fristons Konzepten folgend postuliert Ciompi, dass Angst, Unsicherheit und emotionale Anspannung vor dem Ausbruch einer Psychose einer klinischen Manifestation von erhöhter freier Energie entsprechen [4]. Auch die Symptome einer Psychose entsprechen demnach einem sich Manifestieren von freier Energie.

Die Auseinandersetzung mit der Realität passiert im Falle einer Psychose basierend auf falschen Annahmen über die Realität, welche wiederum auf einer fehlerhaften Wahrnehmung basieren [8]. In dieser Konzeption des Manifestwerdens einer Psychose spielt das Sichaufschaukeln emotionaler Anspannungen eine entscheidende Rolle. Maßnahmen zum Umgang mit der erhöhten affektiven Spannung bekommen in therapeutischen Interventionen einen zentralen Stellenwert, besonders gewinnt die zeitliche Platzierung der Intervention an Bedeutung.

Eine bewusste Gestaltung der therapeutischen Umgebung (sicher, stabil, transparent) kommt bspw. in integrativen Therapiekonzepten zur Anwendung [9, 10]. Das Ziel ist es mitunter, die Vorhersagbarkeit des Verhaltens der Umgebung bei PatientInnen zu verbessern, um damit „ver-rückte“ alternative Erklärungen unnötig zu machen.

Affektregulation passiert bei Frau C durch projektive Identifizierung und in Allmachtsfantasien - ihre als ohnmächtig empfundene Situation und der in der Gesprächssituation drohende Kontrollverlust werden dadurch für eine kurze Weile erträglicher. Für die Explorierende besteht in dieser Situation die Schwierigkeit, sich einzufühlen und die Wirkungen der eigenen Intervention vorsichtig abzuschätzen.

\section{) Die therapeutische Affektverarbeitung bessert auch die Selbstregulationsfähigkeit}

Bei der oben beschriebenen Intervention ist dies nicht gelungen. Die projektive Identifizierung der Patientin wird in nicht verarbeiteter Form unvermittelt zurückgeworfen, was zu einer fortschreitenden Fragmentierung führt.

Vorhersehbar ist in dieser Konstellation für die Gesprächsführende jedenfalls, dass die Art und Weise wie Frau C in ihrer Psychose die Wirklichkeit wahrnimmt und interpretiert sowie wie diese Wahrnehmung von jener der Gesprächsführenden stark abweicht. Die überschießenden Affekte und die Schwierigkeit der Regulation dieser Affekte wahrzunehmen, kann hier der entscheidende Schritt für das Setzen einer geeigneten Intervention sein. Gemäß dem Prinzip des Holdings und Containments liegt eine therapeutische Kompetenz in der „Rêverie“, dem therapeutischen Ahnungsvermögen, welches ein Aufnehmen und Verarbeiten der Affekte des Patienten ermöglicht, um sie in veränderter und erträglicher Form dem Gegenüber wieder zurückzugeben [11]. Diese therapeutische Fähigkeit verbessert also in vielen Interaktionen ebenfalls die Interpretierbarkeit der Umgebung und reduziert gewissermaßen für PatientInnen die freie Energie. Über den inneren Zustand von Frau C lässt sich aus ihrem Verhalten schließen, psychopraxis. neuropraxis $2022 \cdot 25$ :

103-106

https://doi.org/10.1007/s00739-022-00783-y

(c) Der/die Autor(en) 2022

D. Steinmair - N. Masic - D. Schwigon H. Löffler-Stastka

Affektregulation und soziale Kognition bei Psychose

Zusammenfassung

Gelingende soziale Interaktionen sind für das Wohlbefinden eine entscheidende Komponente; Sozialität ist ein Merkmal menschlicher Gruppen. Psychose und Begegnung im sozialen Umfeld stellen eine Herausforderung an die Beziehungsgestaltung dar. Subtile Interventionen von TherapeutInnenseite können eine erfolgreiche Interaktion bewirken. Entscheidend ist die Berücksichtigung der Affektregulation.

\section{Schlüsselwörter}

Wahrnehmung · Affektive Spannung . Holding · Containment - Empathische Mentalisierungsfähigkeit

\section{Affect Regulation and Social Cognition in Psychosis}

\section{Abstract}

Successful social interactions are a crucial component of well-being; sociality is a feature of human groups. Psychosis and encounters in the social environment pose a challenge to relationship building. Subtle interventions from the therapist's side can bring about successful interaction. Consideration of problems with affect regulation is crucial.

\section{Keywords}

Perception - Affective tension · Holding · Containment $\cdot$ Empathic mentalization

dass der Angst vor innerer Vernichtung nichts ausreichend entgegengesetzt werden kann und wurde.

Folgt man psychoanalytischen Erklärungsversuchen, dann konnte bei Frau C ein "gutes inneres Objekt" [12-14] nicht etabliert werden bzw. ist es im Zustand extremer innerer Anspannung nicht zugänglich. Innere Objekte sind mentale Strukturen, welche mentalen Repräsentanzen aus integrierten frühen Beziehungen entsprechen und denen bei der Einordnung neuer Wahrnehmungen, bei der Gestaltung neuer Beziehungen eine ent- 
scheidende Funktion zukommt, wobei sie autonomiefördernd wirken [15]. Die (paranoiden) Ängste werden nach außen projiziert und in der therapeutischen $\mathrm{Be}$ ziehung ausagiert; die „normale“ dynamische Dialektik zwischen mentalen internalisierten Repräsentanzen und den aktuellen äußeren Objekten ist gestört. Dies ist dann der Fall, wenn klare Abgrenzungen zwischen internalisierten Repräsentanzen des Selbst und von andern („Objekten“; [13]) fehlen.

In einer therapeutischen Beziehung geschieht eine bewusste Gestaltung der Beziehung zwischen TherapeutIn und PatientIn. Die Erfahrung, welche eine therapeutische Beziehung hinsichtlich Affektregulation für Patienten bieten kann, ist, das Ausmaß des im eigenen Inneren Aushaltbaren in vielen Interaktionen langsam zu erweitern und dabei neue Strategien der Bewältigung $\mathrm{zu}$ etablieren. TherapeutInnen dienen hierbei zunächst als Erweiterung der Psyche der Psychosebetroffenen. In affektiv aufgeladenen Situationen, wie es auch die empfundene Nähe in einer Therapie bereits darstellt, ist dies eine besondere Herausforderung. Die therapeutische Affektverarbeitung bessert auch die Selbstregulationsfähigkeit, was sich wiederum auf die Wahrnehmung des Selbst in Interaktion mit anderen auswirkt und $\mathrm{zu}$ adäquateren Vorhersagen über die Wirklichkeit führt. Die Vernichtungsangst der Patientin zu beschreiben, damit aufzunehmen, sich durch analytikerzentrierte Interventionen $[16,17]$ als Gegenüber anzubieten und die Bereitschaft zu gemeinsamer Bearbeitung der existenzbedrohenden Ängste auszuloten, kann eine Möglichkeit darstellen.

Soziale Interaktion ist je nach kognitivem Ausgangsfunktionsniveau unterschiedlich erschwert, da das Repertoire auch an kognitiven Strategien sich besonders in solchen affektiv aufgeladenen Situationen schneller erschöpft. Ein Perspektivenwechsel sowie ein Mentalisieren der Zustände des Gegenübers gelingt kaum, wenn die Grenzen zwischen mentalem Selbst und Objektrepräsentanzen, zwischen internalisierten Objektvorstellungen und äußeren Objekten verschwimmen und das psychische Existie-
Hier steht eine Anzeige. 를 Springer 
ren des Ichs jeweils in dem Ausmaß bedroht wird, wie das Objekt durch Projektionen als bedrohlich wahrgenommen wird. Können positive und negativ aufgeladene Repräsentanzen nicht integriert werden (Spaltung), ist dies erwartungsgemäß regelmäßig der Fall.

\section{Fazit für die Praxis \\ - In der Rehabilitation von Patient- Innen mit Psychose ist das Fehlen von psychotischen Symptomen für eine Normalisierung des psychosozialen Funktionsniveaus nicht ausreichend. \\ - Wenn reife Strategien zur Regulie- rung intensiver negativer Affekte fehlen, fällt dem Therapeuten/der Therapeutin die Rolle zu, diese un- erträglichen „Abfallprodukte" einst- weilen zu "containen" und in etwas für den/die PatientIn Erträglicheres zu metabolisieren. \\ - Maßnahmen zur Verbesserung der sozialen Fähigkeiten sind ein mögli- cher Ansatz, um den Outcome positiv zu beeinflussen. Der Emotionsregu- lation kommt in diesem Kontext eine entscheidende Bedeutung zu.}

\section{Korrespondenzadresse

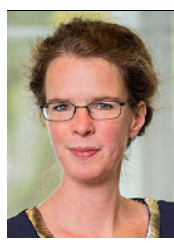 \\ Univ. Prof. Dr. med. univ. H. Löffler-Stastka Klinik für Psychoanalyse und Psychotherapie, Medizinische Universität Wien Währinger Gürtel 18-20, 1090 Wien, Österreich henriette.loeffler- stastka@meduniwien.ac.at}

Funding. Open access funding provided by Medical University of Vienna.

\section{Einhaltung ethischer Richtlinien}

Interessenkonflikt. D. Steinmair, N. Masic, D. Schwigon und H. Löffler-Stastka geben an, dass kein Interessenkonflikt besteht

Für diesen Beitrag wurden von den Autoren keine Studien an Menschen oder Tieren durchgeführt. Für die aufgeführten Studien gelten die jeweils dort angegebenen ethischen Richtlinien.

Open Access. Dieser Artikel wird unter der Creative Commons Namensnennung 4.0 International Lizenz veröffentlicht, welche die Nutzung, Vervielfältigung
Bearbeitung, Verbreitung und Wiedergabe in jeglichem Medium und Format erlaubt, sofern Sie den/die ursprünglichen Autor(en) und die Quelle ordnungsgemäß nennen, einen Link zur Creative Commons Lizenz beifügen und angeben, ob Änderungen vorgenommen wurden.

Die in diesem Artikel enthaltenen Bilder und sonstiges Drittmaterial unterliegen ebenfalls der genannten Creative Commons Lizenz, sofern sich aus der Abbildungslegende nichts anderes ergibt. Sofern das betreffende Material nicht unter der genannten Creative Commons Lizenz steht und die betreffende Handlung nicht nach gesetzlichen Vorschriften erlaubt ist, ist für die oben aufgeführten Weiterverwendungen des $\mathrm{Ma}$ terials die Einwilligung des jeweiligen Rechteinhabers einzuholen.

Weitere Details zur Lizenz entnehmen Sie bitte der Lizenzinformation auf http://creativecommons.org/ licenses/by/4.0/deed.de.

\section{Literatur}

1. Wirtz MA (2021) Soziale Kognition. Lexikon de Psychologie, 20., aktualisierten Aufl. Hogrefe, Göttingen

2. Lexikon der Psychologie Soziale Kognition. https:// www.spektrum.de/lexikon/psychologie/sozialekognition/14518.Zugegriffen: 1. Jan. 2022

3. de Sousa P, Sellwood W, Griffiths M, Bentall RP (2019) Disorganisation, thought disorder and socio-cognitive functioning in schizophrenia spectrum disorders. Br J Psychiatry 214(2):103-112. https://doi.org/10.1192/bjp.2018.160

4. Ciompi L, Tschacher W (2021) Affect-logic, embodiment, synergetics, and the free energy principle: new approaches to the understanding and treatment of schizophrenia. Entropy (Basel) 3(12):1619. https://doi.org/10.3390/e23121619

5. Carrión RE, Walder DJ, Auther AM, McLaughlin D, Zyla HO, Adelsheim S, Calkins R, Carter CS, McFarland B, Melton R, Niendam T, Ragland JD, Sale TG, Taylor SF, McFarlane WR, Cornblatt BA (2018) From the psychosis prodrome to the firstepisode of psychosis: no evidence of a cognitive decline. J Psychiatr Res 96:231-238. https://doi. org/10.1016/j.jpsychires.2017.10.014

6. Kharawala S, Hastedt C, Podhorna J, Shukla H, Kappelhoff B, Harvey PD (2021) The relationship between cognition and functioning in schizophrenia: a semi-systematic review. Schizophr Res Cogn 27:100217. https://doi.org/10.1016/j.scog.2021. 100217

7. Friston K(2010) The free-energy principle: a unified brain theory? Nat Rev Neurosci 11(2):127-138. https://doi.org/10.1038/nrn2787

8. Tschacher W, Giersch A, Friston KJ (2017) Embodiment and schizophrenia: a review of implications and applications. Schizophr Bull 43:745-755

9. Mosher LR, Menn AZ (1978) Community residentia treatment for schizophrenia: two-year follow-up data. HospCommunity Psychiatry 29(11):715-723. https://doi.org/10.1176/ps.29.11.715

10. Ciompi L, Hoffmann H (2004) Soteria Berne. An innovative milieu therapeutic approach to acute schizophrenia based on the concept of affect-logic World Psychiatry 3:140-146

11. Bion WR (1962) Learning from experience. Karnac Books, London

12. Klein M (2001) Das Seelenleben des Kleinkindes und andere Beiträge zur Psychoanalyse. KlettCotta, Stuttgart
13. Rubens RL (1994) Fairbairn's structural theory. In: Grotstein JS, Rinsley DB (Hrsg) Fairbairn and the origins of object relations. Guilford, New York

14. Fairbairn WRD (2007) Das Selbst und die inneren Objektbeziehungen. Eine psychoanalytische Objektbeziehungstheorie. Psychosozial-Verlag Gießen

15. Stierlin H (1971) Die Funktion innerer Objekte Psyche 25(2):81-99

16. Steiner J (1997) Problems of psychoanalytic technique: patient centered and analyst-centered interpretations. In:Schafer R(Hrsg) The contemporary Kleinians of London. International Universities Press, Madison, S372-392

17. Löffler-Stastka H, Blueml V, Boes C (2010) Exploration of personality factors and thei predictive impact on therapy utilization: the externalizing mode of functioning. Psychother Res 20(3):295-308. https://doi.org/10.1080/ 10503300903436710

Hinweis des Verlags. Der Verlag bleibt in Hinblick auf geografische Zuordnungen und Gebietsbezeichnungen in veröffentlichten Karten und Instituts adressen neutral. 